\title{
Tramadol-Induced Hiccups: A Report of Two Cases
}

\author{
Radhika Panchal $^{1} \cdot$ Vishal Bhutt $^{2} \cdot$ Ashish Anovadiya ${ }^{1} \cdot$ Bhargav Purohit $^{1}$. \\ Firdaus Dekhaiya $^{2} \cdot$ Nutanbala Goswami $^{1}$
}

\begin{abstract}
Hiccups can be idiopathic, psychogenic and organic, with drugs being one of the most important causes of hiccups. Although the exact pathophysiological processes involved are still poorly understood, the neurotransmitters dopamine, serotonin, and $\gamma$-aminobutyric acid (GABA) have been documented to play a significant role in the generation of hiccups. We report on two patients with cellulitis who developed hiccups with the use of tramadol as an analgesic. The possible mechanisms and clinical implications of this rare adverse event are discussed. Both patients recovered from the hiccups with the use of baclofen tablets.
\end{abstract}

Radhika Panchal

drradhikapanchal@yahoo.com

Vishal Bhutt

drvishalbutt@yahoo.com

Ashish Anovadiya

drashishanovadiya@yahoo.com

Bhargav Purohit

purohitbhargav@rediffmail.com

Firdaus Dekhaiya

firdausd2001@yahoo.com

Nutanbala Goswami

drnutanbalagoswami78@gmail.com

1 Department of Pharmacology, Government Medical College and Sir Takhtasinhji General Hospital, 1st floor, Bhavnagar, Gujarat 364001, India

2 Department of Surgery, Government Medical College and Sir Takhtasinhji General Hospital, Bhavnagar, Gujarat 364001, India

\section{Key Points}

Hiccups due to tramadol use are a rare entity.

If not treated, hiccups can lead to serious complications.

In both patients in this case report, hiccups were successfully treated with baclofen.

\section{Introduction}

Hiccups (singultus) are the involuntary and rapid expulsion of air from the lungs with the asynchronous closure of the glottis, causing disruption of the flow of air. They can be acute $(<48 \mathrm{~h})$, enduring $(>48 \mathrm{~h})$ or persistent ( $>1$ month) [1]. Hiccups caused by distention of the stomach, stress, rapid change in temperature, and alcohol intake are generally benign and self-limiting. In the US, approximately 4000 hospital admissions are due to hiccups each year [2], and the incidence is higher in males aged 50 years and older [3]. Persistent hiccups can disturb sleep patterns and daily activities of patients. They can be due to an underlying pathology or can have an organic cause, and, if left untreated, can be serious. Hiccups are modulated by a reflex arc with afferents, including the vagus, phrenic nerve and sympathetic chain; a central hiccup center, including the respiratory center, phrenic nerve nuclei, and reticular part of the hypothalamus; and efferent, which includes the phrenic nerve, glottis, and nerves to the intercostal muscles [1]. Drug-induced hiccups are a rare entity and diagnosis 
requires exclusion of other causes. Corticosteroids, benzodiazepines, sulfonamides, opioids, cisplatin, and dopamine are common causative agents.

Tramadol is a centrally acting opioid analgesic that is indicated for acute and chronic pain, such as pain due to surgery or trauma and malignant diseases. Nausea, vomiting, constipation, sweating, dry mouth, headache, lower leg edema, swelling in the face or tongue, rhinorrhoea, dry skin, and burning in the eyes are the most frequently occurring adverse drug reactions. Respiratory depression only occurs when high doses of tramadol, or concomitant drugs with centrally inhibiting effects, are used [1]. However, the incidence of tramadol-induced hiccups is rare. We report two cases of cellulitis admitted to a surgical ward at Sir Takhtasinhji General Hospital, Bhavnagar, Gujarat, India, who were treated with tramadol and who developed hiccups that responded well to baclofen and tramadol withdrawal. Written informed consent was received from both patients.

\section{Case 1}

A 61-year-old male complained of severe pain in the right lower limb. On local examination, the limb was edematous, red and swollen, and with blisters and ulcers; bilateral popliteal and pedal pulse were also present. Vitals were normal and there was no history of insect bites or drug allergies, although there was a past history of diabetes mellitus since 20 years of age. There was no clinical evidence of gastrointestinal, central nervous system, cardiovascular, renal (uremia) or metabolic disorder and brain trauma. Investigations revealed the following: random blood sugar (RBS) test, $232 \mathrm{mg} / \mathrm{dl}$; serum creatinine, $1.7 \mathrm{mg} / \mathrm{dl}$. A provisional diagnosis of right lower limb cellulitis was made. Surgical wound debridement of the right lower limb was performed under spinal anesthesia on the same day. On admission, the patient was administered injectable amoxicillin $1.2 \mathrm{~g}$ intravenously 8-hourly, omeprazole $20 \mathrm{mg}$ capsules intravenously 12-hourly, injectable tramadol $100 \mathrm{mg}$ intravenously 12-hourly, and injectable Actrapid 4 IU subcutaneously (as per the RBS test). After $1 \mathrm{~h}$, the patient developed hiccups. The patient drank water frequently to try and relieve the hiccups, but they continued without a pause for $1 \mathrm{~h}$. On day 2, the patient was switched to tramadol $50 \mathrm{mg}$ oral tablets 12-hourly, and the remaining treatment was continued as per the first day. On day 2, the patient again developed hiccups $1 \mathrm{~h}$ after taking a tablet, which continued for $2 \mathrm{~h}$ without a pause. The patient had a bloating sensation in his stomach after taking the tramadol tablet.

The patient tried non-pharmacological measures to try and relieve the hiccups, such as leaning forward and forceful air blowing, but there was no relief. After $2 \mathrm{~h}$, the hiccups subsided on their own, but started again half an hour after intake of the evening dose of tramadol, and continued into the night. On day 3 , and using the same treatment approach, the patient again developed hiccups, which were hampering sleep patterns and daily activities. On day 4, the patient developed hiccups within $15 \mathrm{~min}$ of drug intake, which continued for $3 \mathrm{~h}$. The patient was confused and tried no pharmacological measures, and the hiccups were not relieved. On day 5, the patient informed his physician about the hiccups. Tramadol was discontinued and a baclofen $10 \mathrm{mg}$ tablet 8-hourly was added to the treatment regimen. The hiccups subsided $3 \mathrm{~h}$ after the withdrawal of tramadol, and the baclofen $10 \mathrm{mg}$ tablet 8-hourly was continued for 3 days; the hiccups did not reappear thereafter.

\section{Case 2}

A 60-year-old male complained of severe burning pain in the right lower limb. On local examination, the right lower thigh was edematous and red, and bilateral popliteal and pedal pulse were present. Vitals were normal and there was no history of insect bites or drug allergies. There was no clinical evidence of gastrointestinal, central nervous system, cardiovascular, renal or metabolic disorder. A provisional diagnosis of right lower limb cellulitis was made. Surgical wound debridement of the right lower limb was performed under spinal anesthesia on the same day, after examination. Investigations conducted on admission revealed the following: RBS test, $298 \mathrm{mg} / \mathrm{dl}$; serum creatinine, $1.44 \mathrm{mg} / \mathrm{dl}$. Hematological investigations conducted revealed hemoglobin, $13.5 \mathrm{~g} / \mathrm{dl}$; total white blood cell count 21,000/cumm.

On admission, the patient was treated with injectable cefoperazone-sulbactam $1.5 \mathrm{~g}$ intravenously 12-hourly, injectable ciprofloxacin $400 \mathrm{mg}$ intravenously 12-hourly, duphalac syrup $20 \mathrm{ml}$ 8-hourly, injectable pantoprazole $40 \mathrm{mg}$ intravenous injection 24-hourly, injectable furosemide $40 \mathrm{mg}$ intravenously in normal saline 12-hourly, tramadol $100 \mathrm{mg} /$ day tablet, and injectable regular insulin 6 IU subcutaneously (RBS 291 g/ dl).

Two hours after taking the tramadol tablet the patient developed hiccups, which continued for $3 \mathrm{~h}$ and then subsided. On day 2, the patient received the same treatment regimen, with the addition of two medications: sorbiline syrup $3 \mathrm{mg} /$ day and metformin $500 \mathrm{mg}$ tablet were administered 12-hourly, and the remaining treatment was continued as above. After receiving a morning dose of tramadol, the patient again developed hiccups. The patient drank cold water frequently to try and relieve the hiccups, 
and they eventually subsided just $1 \mathrm{~h}$ before the patient received the next evening dose of tramadol. One hour after receiving the evening dose, the patient again developed hiccups, which continued for the rest of the day. The patient had a bloating sensation in his stomach after taking the tramadol tablet. On days 3, 4 and 5, the same treatments were continued, and hiccups developed $1 \mathrm{~h}$ after each dose. During this period, the patient had tried non-pharmacological measures, with the thought that the hiccups would gradually subside on their own in time. On day 6 , the patient developed constant severe hiccups that lasted for more than $8 \mathrm{~h}$ and continued for the entire night. The physician was finally informed on the seventh day. Tramadol was withdrawn and injectable metoclopramide $10 \mathrm{mg}$ intravenously 12 -hourly and baclofen $10 \mathrm{mg}$ tablet 8-hourly for 3 days were administered to relieve the hiccups. The hiccups subsided and have not since reappeared.

\section{Discussion}

Both cases show a strong temporal association of hiccups with tramadol as the hiccups started after tramadol intake and prompt relief occurred upon cessation of the drug. Based on the WHO's causality assessment scale [9], reactions were categorized as certain. Both cases were mild in severity and definitely preventable as per the modified Hartwig and Siegel severity scale and modified Schumock and Thornton scale, respectively.

Although the exact mechanism by which hiccups develops is poorly understood, a central component of the hiccups reflex arc is situated in the medulla. Serotonin, dopamine, $\gamma$-aminobutyric acid (GABA), glutamate, and glycine neurotransmitters can regulate this central mechanism of hiccups [1]. This reflex arc is also regulated by input from catecholaminergic and serotonergic afferents. Tramadol is a racemate of two enantiomers. The cisenantiomer binds to the $\mu$-opiate receptor and inhibits the serotonin reuptake. Inhibition of serotonin reuptake by tramadol can lead to stimulation of the central component of the reflex arc, while stimulation of opioid receptors decreases GABA release, and hence increases dopamine release. Although tramadol has less affinity to the $\mu$-opioid receptor, its major active metabolite O-desmethyltramadol has greater affinity to the $\mu$-opioid receptor [4]. The transenantiomer of tramadol inhibits norepinephrine reuptake and stimulates $\alpha 2$ receptors [5]. Therefore, increased catecholamine due to reuptake inhibition can also stimulate the reflex arc of hiccups and can lead to skeletal muscle contractions, including the diaphragm [6]. The positive dechallenge and positive accidental rechallenge in both cases makes the causal relationship between tramadol and hiccups stronger. Other possible mechanisms can be distension in the stomach, which can result in vagus nerve irritation, and hence afferent impulses of hiccups are activated. In both cases mentioned above, the patient had complained of a bloating sensation in his stomach after intake of tramadol [7].

\section{Conclusions}

Hiccups are generally considered non-specific and not as a consequence of a disease or a drug being taken by a patient, therefore less attention is paid to this issue. As in these two cases, the patient failed to inform their physician until the hiccups became severe. Such a delay and such persistent hiccups may cause significant negative intrathoracic pressure, which may result in hypotension, bradycardia, pneumomediastinum, and subcutaneous emphysema, and, rarely, bradyarrhythmias [8]. Healthcare professionals should be aware of the possible relationship between hiccups and tramadol, especially while treating a patient with no underlying complications.

Acknowledgements The authors would like to thank Mr. Bhavesh Chavada, Pharmacovigilance Technician Associate, Department of Pharmacology, Government Medical College, Bhavnagar, Gujarat, India.

Author Contributions Dr. Radhika Panchal conducted the literature review and wrote the case description, abstract, discussion, and conclusions. Dr. Ashish Anovadiya assisted with the literature search review and scrutiny of the case report, provided guidance in writing the discussion, and performed overall drafting of the case report. Dr. Vishal Bhutt assisted in procuring the case report and writing the case description, and assisted with treatment guidance. Dr. Bhargav Purohit assisted with writing the case discussion and overall drafting of the case report. Dr. Firdaus Dekhaiya assisted with procuring the case report and identification. Dr. Nutanbala Goswami assisted with the literature review.

\section{Compliance with Ethical Standards}

Funding No sources of funding were used to assist in the preparation of this case report.

Conflict of interest Radhika Panchal, Ashish Anovadiya, Vishal Bhutt, Bhargav Purohit, Firdaus Dekhaiya and Nutanbala Goswami have no conflicts of interest to declare that are directly relevant to the content of this case report.

Patient consent Written informed consent was obtained from both patients prior to publication of this case report. A copy of the written consents may be requested for review from the corresponding author.

Open Access This article is distributed under the terms of the Creative Commons Attribution-NonCommercial 4.0 International License (http://creativecommons.org/licenses/by-nc/4.0/), which permits any noncommercial use, distribution, and reproduction in any medium, provided you give appropriate credit to the original author(s) and the source, provide a link to the Creative Commons license, and indicate if changes were made. 


\section{References}

1. Nausheen F, Mohsin H, Lakhan SE. Neurotransmitters in hiccups. Springerplus. 2016;5:1357.

2. Dobelle WH. Use of breathing pacemakers to suppress intractable hiccups of up to thirteen years' duration. ASAIO J. 1999;45(6):524-5.

3. Cymet TC. Retrospective analysis of hiccups in patients at a community hospital from 1995-2000. J Natl Med Assoc. 2002;94(6):480-3.

4. Sibell DM, Colantonio AJ, Stacey BR. Successful use of spinal cord stimulation in the treatment of severe Raynaud's disease of the hands. Anesthesiology. 2005;102(1):225-7.
5. Ezzeldin E, Souror WA, El-Nahhas T, Soudi AN, Shahat AA. Biochemical and neurotransmitters changes associated with tramadol in streptozotocin-induced diabetes in rats. Biomed Res Int. 2014;2014:238780.

6. Bowman WC, Raper C. The effects of adrenaline and other drugs affecting carbohydrate metabolism on contractions of the rat diaphragm. Br J Pharmacol Chemother. 1964;23:184-200.

7. Aruna B, Theophilus B. Acute distension of abdomen secondary to tramadol. Pharmacol Online. 2010;1:7-8.

8. Suh WM, Krishnan SC. violent hiccups: an infrequent cause of brady arrhythmias. West J Emerg Med. 2009;10(3):176-7.

9. Zaki S. Adverse drug reaction and causality assessment scales. Lung India. 2011;28(2):152. 